\title{
High-throughput sequencing analysis of the microbial community in coastal intensive mariculture systems
}

\author{
Jian-Hua Wang ${ }^{\mathrm{a}}$, Jian $\mathrm{Lu}^{\mathrm{a}, \mathrm{b}, *}$, Yu-Xuan Zhang ${ }^{\mathrm{a}, \mathrm{b}}$, Jun Wu ${ }^{\mathrm{b}, \mathrm{c}}$, Cui Zhang ${ }^{\mathrm{a}, \mathrm{b}}$, Xiaobin Yu ${ }^{\mathrm{a}, \mathrm{b}}$, \\ Zhenhua Zhang ${ }^{\mathrm{d}}$, Hao Liu ${ }^{\mathrm{e}}$, Wen-Hao Wangf \\ ${ }^{a}$ Key Laboratory of Coastal Environmental Processes and Ecological Remediation, Yantai Institute of Coastal Zone Research, Chinese Academy of Sciences, Yantai, \\ Shandong 264003, People's Republic of China \\ ${ }^{\mathrm{b}}$ University of Chinese Academy of Sciences, Beijing, 100049, People's Republic of China \\ c Qinghai Institute of Salt Lakes, Chinese Academy of Sciences, Xining, Qinghai 810008, People's Republic of China \\ d School of Resources and Environmental Engineering, Ludong University, Yantai, Shandong 264025, People's Republic of China \\ e Shandong Oriental Ocean Sci-tech Co. Ltd, Yantai, Shandong 264003, People's Republic of China \\ ${ }^{\mathrm{f}}$ Yantai Fisheries Research Institute, Yantai, Shandong 264003, People's Republic of China
}

\section{A R T I C L E I N F O}

\section{Keywords:}

High-throughput sequencing

Microbial community

Conventional mariculture system

Recirculating mariculture system

\begin{abstract}
A B S T R A C T
The conventional and recirculating mariculture systems are two typical intensive mariculture systems in the coastal zone. This study used high-throughput sequencing method to investigate the structural profiles of microbial communities in conventional and recirculating mariculture farms. The results showed that 13,842 OTUs (operational taxonomic units) were detected at a similarity level of $97 \%$. Conventional and recirculating systems exhibited significant difference in microbialcommunity based on the results of the taxonomy and relative abundance of bacteria. Among the top 10 genera in coastal intensive mariculture systems, the predominant bacteria in the conventional intensive mariculture system were Pseudofulvibacter, Polaribacter, Glaciecola, Crocinitomix, Colwellia, Oleispira, and Balneola, while those in the recirculating systems were Vibrio, Alteromonas, and Pseudoalteromonas. Bacterial communities of different fish ponds suggested that the bacterial groups exhibited fish-specific or water treatment stage-specific features. Potential pathogens such as Vibrio, Arcobacter, Pseudoalteromonas, and Shewanella were readily accumulated as dominant bacteria in the recirculating mariculture system. The detection of similar potential pathogens in both the mariculture systems and the adjacent coastal water indicate that the mariculture farms without recirculating system could be the hotspots of pathogens and have great influences on the surrounding coastal environment. The wastewater treatment units used in recirculating farm could remove bacteria effectively, suggesting that the recirculating mariculture system may be more environmentally friendly than the conventional mariculture system.
\end{abstract}

\section{Introduction}

Aquaculture is one of the fastest growing food-producing sectors (FAO, 2009), with the products constituting an important food supply. The global aquaculture industry is dominated by Asian countries such as China that account for approximately $71 \%$ of the total global aquaculture production (Sapkota et al., 2008). Mariculture, one of the most important aquaculture in the coastal zone, has become more intensive in recent years (Gao et al., 2012). However, the rapid development in the mariculture industry has exerted significant environmental influence on coastal ecosystems. These influences include eutrophication (Holmer et al., 2005; Kalantzi and Karakassis, 2006), chemical pollutions (Antunes and Gil, 2004; Cabello, 2006; Sapkota et al., 2008), disturbed benthic fauna communities (Vezzulli et al. 2008; Tomassetti et al., 2009), and changes in the biodiversity and community structures of bacteria (Gao et al., 2012; Harnisz et al., 2015; Muziasari et al., 2017). The distribution of microorganisms in the aquaculture system is important for marine fish culture because both pathogens and probiotics can profoundly impact the development and physiological function of aquaculture organisms (Rungrassamee et al., 2016; Viljamaa-Dirks, 2016; Xiong et al. 2016). The wide use of antibiotics in the aquaculture leads to wide-spreading antibiotic resistant pathogens and the changes in structures of bacterial community in culture systems (Xiong et al., 2015; Muziasari et al., 2017). As a result, it is important to study the

\footnotetext{
* Corresponding author at: Key Laboratory of Coastal Environmental Processes and Ecological Remediation, Yantai Institute of Coastal Zone Research, Chinese Academy of Sciences, Yantai 264003, People's Republic of China.

E-mail address: jlu@yic.ac.cn (J. Lu).
} 
microbial community in the mariculture systems of the coastal zone and evaluate the environmental impact of the fish farms.

High-throughput sequencing is the most advanced technique to study microbial diversity and community at present (Xiong et al., 2015; Zheng et al., 2017) because it can detect most of the species in the tested sample without culturing. The high-throughput sequencing method based on 16S rDNA amplicon sequencing technology can make an in-depth analysis on the bacteria community and abundance (Leite et al., 2012). The amplicon sequencing approach has been widely used to characterize the microbial communities and abundances in various environments such as rivers (Zhou et al., 2017), aquaculture environment (Zheng et al., 2017), and soil (Hua et al., 2017).

The aquaculture industry covers a wide range of units from simple traditional systems to integrated aquaculture-agriculture systems (Heuer et al., 2009). The conventional (the flow-through system) and recirculating mariculture systems are two typical mariculture systems intensively used in the coastal zone. The mariculture wastewater is circulated and recycled after a systemic treatment in the recirculating system while the wastewater in the conventional system is discharged directly (or with simple treatment) into the sea (Su et al., 2011; Xiong et al., 2015). Mariculture is a major industry in global coastal zones and has led to concerns regarding the impact of fish farms on the coastal environment. Limited information is available on investigating the difference of the microbial community between the conventional and recirculating mariculture systems. The objectives of the present study are to compare the microbial communities of the conventional and recirculating mariculture systems using high-throughput sequencing technique, and evaluate the influences of the intensive mariculture on coastal aquatic environment based on the impacts of microbial community especially potential pathogen.

\section{Materials and methods}

\subsection{Sample collection}

The water samples were collected from two intensive mariculture systems with or without recirculating units in Yantai City which is the most important mariculture city in China. The production of intensive seawater farming in Yantai City was 28,000 tons, accounting for 1/6 of China in 2015 (Municipal Work Report of Yantai, 2015). The mariculture systems and the sampling points were shown in Fig. 1. Fig. 1a showed the conventional intensive mariculture system ( $\mathrm{ZZ}$ farm) without recirculating unit while Fig. 1b illustrated the recirculating mariculture system (DF farm). The Ponds 1, 2, 3, and 4 in ZZ farm are for Penaeus vannamei, Scophthalmus maximus, fish-fry of $S$. maximus, and Platichthys stellatus, respectively. Fish pond in DF farm is for Salmo salar.
The recirculation ration (the proportion of the water reclaimed to the water needed for the breeding pond) is $90 \%$. The numbers (1) (2) (3) (4)(5)(6) (7) denote the sampling points. The water samples collected from the sampling points of the conventional system ( $\mathrm{ZZ} \mathrm{farm)}$ were marked as ZZ1, ZZ2, ZZ3, ZZ4, ZZ5, ZZ6 and ZZ7, respectively. The samples ZZ1, ZZ6, and ZZ7 referred to the influent, the stabilization and coastal water sample, respectively while ZZ2, ZZ3, ZZ4, and ZZ5 referred to the sample of fish pond $1,2,3$, and 4 , respectively. The water samples collected from the sampling points of the recirculating system (DF farm) were marked as DF1 (from the influent), DF2 (from fish pond), DF3 (from protein separator), DF4 (from biochemical pool for wastewater treatment), DF5 (from biological aerobic pool for wastewater advanced treatment), and DF6 (from the recycled water), respectively. Water samples (30 L/point) were filtered through $0.22 \mu \mathrm{m}$ micropore membrane and the filtered materials were kept at $-80^{\circ} \mathrm{C}$ for further study.

\subsection{DNA extraction, library preparation and sequencing}

\subsubsection{Extraction of genome DNA}

The filtered samples were transported on dry-ice to Novegene (Beijing, China) for DNA extraction and sequencing. The total genomic DNA was extracted by CTAB/SDS method (Armougom and Raoult, 2009). The concentrations and purity of DNA were monitored on $1 \%$ agarose gels. DNA was diluted to $1 \mathrm{ng} / \mu \mathrm{L}$ using sterile water according to the concentration.

\subsubsection{PCR amplification}

16S rRNA genes of distinct regions 16S V4-V5 were amplified using the universal forward 515 F (5'-GTGCCAGCMGCCGCGG-3') and reverse 907R ( $5^{\prime}$-CCGTCAATTCMTTTRAGTTT-3') (Armougom and Raoult, 2009) with the barcode (listed in Table 1). All PCR reactions were carried out with Phusion ${ }^{\circledR}$ High-Fidelity PCR Master Mix (New England Biolabs). The PCR reaction system $(25 \mu \mathrm{L})$ contained $17.2 \mu \mathrm{L} \mathrm{ddH}_{2} \mathrm{O}$, $2.5 \mu \mathrm{L} 10 \times$ PCR buffer, $2.5 \mathrm{mM}$ dNTP mixture, $1 \mu \mathrm{L}$ of each primer, 1.5 $\mathrm{U}$ of Taq DNA polymerase, and $1 \mu \mathrm{L}$ of template DNA. The PCR cycling procedures were the following: $2 \mathrm{~min}$ at $95^{\circ} \mathrm{C}$, followed by 25 cycles ( $95^{\circ} \mathrm{C}$ for $30 \mathrm{~s}, 55^{\circ} \mathrm{C}$ for $30 \mathrm{~s}$, and $72{ }^{\circ} \mathrm{C}$ for $30 \mathrm{~s}$ ) and a final extension at $72^{\circ} \mathrm{C}$ for $8 \mathrm{~min}$, and then held at $4{ }^{\circ} \mathrm{C}$. The PCR products were checked on $2 \%$ agarose gel. Samples with bright main strip between $400-450$ bp were chosen for further experiments. The target PCR products were mixed in equidensity ratios. Finally, mixed PCR products were purified with Qiagen Gel Extraction Kit (Qiagen, Germany).

\subsubsection{Library preparation and sequencing}

Sequencing libraries were generated using TruSeq DNA PCR-free

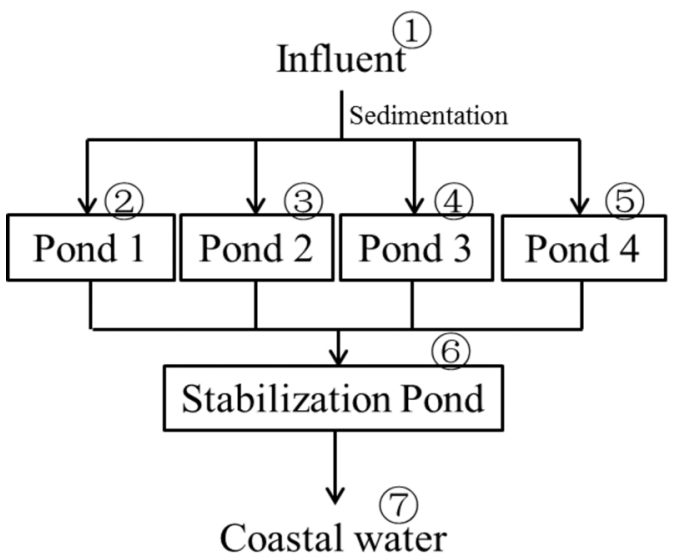

a. ZZ farm

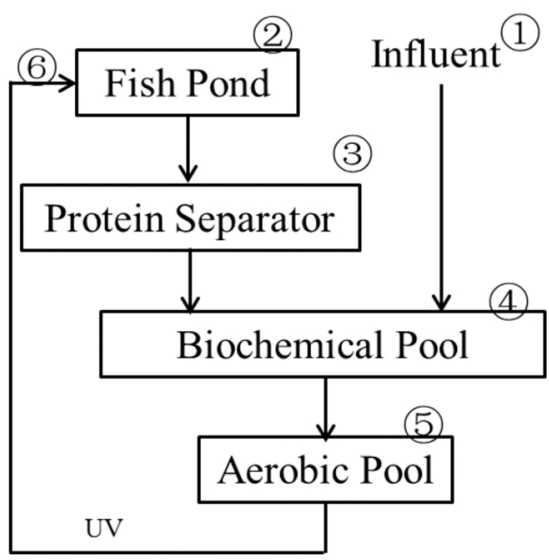

Fig. 1. Flowchart and sampling points of the two fish farms: (a) ZZ, a conventional mariculture system; (b) DF, a recirculating mariculture system. The pond 1 in $\mathrm{ZZ}$ farm is for Penaeus vannamei, pond 2 is for Scophthalmus maximus, pond 3 is for fish-fry of $S$. maximus, and pond 4 is for Platichthys stellatus. The fish pond in DF farm is for Salmo salar. The recirculation ration is $90 \%$. The numbers (1)(2)(3)(4)(5)(6) (7) are for the sampling points. 
Table 1

Barcodes used in this study.

\begin{tabular}{ll}
\hline Sample & Barcode Sequence \\
\hline ZZ1 & AACGTGAT, GGTGCGAA \\
ZZ2 & AACGTGAT, GTCGTAGA \\
ZZ3 & AACGTGAT, TCTTCACA \\
ZZ4 & AACGTGAT, TTCACGCA \\
ZZ5 & AACGTGAT, AATCCGTC \\
ZZ6 & AACGTGAT, AATGTTGC \\
ZZ7 & AACGTGAT, AGCACCTC \\
DF1 & AACGTGAT, ATAGCGAC \\
DF2 & AACGTGAT, CCTCTATC \\
DF3 & AACGTGAT, CGGATTGC \\
DF4 & AACGTGAT, GAGTTAGC \\
DF5 & AACGTGAT, GATGAATC \\
DF6 & ACAGCAGA, GGTGCGAA
\end{tabular}

Note: In the conventional mariculture system (ZZ farm), samples ZZ1, ZZ6, and ZZ7 refer to the influent, the stabilization and coastal water sample, respectively while $Z Z 2$, ZZ3, ZZ4, and ZZ5 refer to the sample of fish pond 1, 2, 3, and 4 , respectively. In the recirculating mariculture system (DF farm), samples DF1, DF2, DF3, DF4, DF5, and DF6 refer to the influent, fish pond, protein separator, biochemical pool for wastewater treatment, biological aerobic pool for wastewater advanced treatment, and the recycled water.

Sample Preparation Kit (Illumina, USA) based on recommendations of manufacture. Index codes were added. The library quality was assessed on the Qubit 2.0 Fluorometer (Thermo Scientific) and Agilent Bioanalyzer 2100 system. The library was finally sequenced on an Illumina HiSeq2500 platform and 250 bp paired-end reads were generated.

\subsection{Data analysis}

\subsubsection{Data filtration}

Paired-end reads were assigned to samples based on their unique barcode and truncated by cutting off the barcode and primer sequence. Paired-end reads were merged using FLASH (V1.2.7). Quality filtering on the raw tags was performed by QIIME (V1.7.0) (Caporaso et al., 2010). The chimera sequences were removed by UCHIME Algorithm (Edgar et al., 2011), and the effective tags were obtained.

\subsubsection{OTU cluster and species annotation}

Sequences analysis was performed by Uparse software (Uparse v7.0.1001). Sequences with similarity $\geq 97 \%$ were assigned to the same OTUs (Armougom and Raoult, 2009). Representative sequence for each OTU was screened for further annotation. In terms of each representative sequence, the GreenGene Database was used based on RDP classifier (Version 2.2) (Wang et al., 2007) algorithm to annotate taxonomic information.

\subsubsection{Phylogenetic relationship construction}

Multiple sequence alignment was conducted using the MUSCLE software (Version 3.8.31) in order to study phylogenetic relationship of different OTUs as well as the difference of the dominant species in different samples (groups).

\subsubsection{Data normalization}

Abundance of OTUs was normalized using a standard of sequence number corresponding to the sample with the least sequences. Subsequent analysis of alpha diversity and beta diversity was performed in the basis of the output normalized data.

\subsubsection{Alpha diversity and beta diversity analysis}

Alpha diversity was calculated with QIIME (Version 1.7.0) and displayed with R software (Version 2.15.3). Beta diversity analysis was
Table 2

Alpha diversity indices and OTUs number of all of the samples.

\begin{tabular}{llllll}
\hline Snormal & OTUs & Observed Species & Shannon $^{\mathrm{a}}$ & Chao $1^{\mathrm{b}}$ & Good's coverage $^{\mathrm{c}}$ \\
\hline ZZ1 & 1653 & 1496 & 6.297 & 1665.783 & 0.993 \\
ZZ2 & 780 & 780 & 5.629 & 1139.841 & 0.993 \\
ZZ3 & 645 & 476 & 3.985 & 649.067 & 0.996 \\
ZZ4 & 657 & 528 & 4.133 & 620.286 & 0.996 \\
ZZ5 & 589 & 464 & 3.247 & 674.348 & 0.995 \\
ZZ6 & 824 & 693 & 5.950 & 783.533 & 0.996 \\
ZZ7 & 982 & 856 & 5.747 & 969.286 & 0.996 \\
DF1 & 1820 & 1732 & 7.694 & 1803.600 & 0.996 \\
DF2 & 1332 & 1191 & 6.501 & 1284.531 & 0.996 \\
DF3 & 878 & 749 & 5.926 & 854.940 & 0.996 \\
DF4 & 900 & 780 & 4.639 & 857.020 & 0.996 \\
DF5 & 1260 & 1104 & 6.702 & 1271.592 & 0.994 \\
DF6 & 1522 & 1350 & 7.285 & 1483.155 & 0.994 \\
\hline
\end{tabular}

Note: In the conventional mariculture system (ZZ farm), samples ZZ1, ZZ6, and ZZ7 refer to the influent, the stabilization and coastal water sample, respectively while ZZ2, ZZ3, ZZ4, and ZZ5 refer to the sample of fish pond 1, 2, 3, and 4, respectively. In the recirculating mariculture system (DF farm), samples DF1, DF2, DF3, DF4, DF5, and DF6 refer to the influent, fish pond, protein separator, biochemical pool for wastewater treatment, biological aerobic pool for wastewater advanced treatment, and the recycled water.

a The Shannon index is used to calculated the diversity of bacteria community.

b The Chao1 estimator is used to estimate the community richness.

c The Good's coverage is used to evaluate the depth of sequencing.

performed by QIIME software (Version 1.7.0) and ggplot2 package in $\mathrm{R}$ software (Version 2.15.3).

The sequences derived from high-throughput sequencing were deposited in the National Center for Biotechnology Information (NCBI) database under accession number SRP128966.

\section{Results and discussion}

3.1. Diversity and abundance of bacteria in coastal intensive mariculture farms

Total 925,227 reads with an average of 56,218 high-quality sequences per sample were obtained from all the 13 samples. These sequences clustered into 13,842 OTUs at a similarity level of $97 \%$. The Good's coverages of all samples were greater than 0.99 , suggesting that the sequencing depth of all samples were sufficient to represent bacterial community in the mariculture environments (Table 2). Shannon diversity index (Magurran, 1988; Chao and Bunge, 2002) showed variations of 3.247-7.694 in these samples (Table 2). The Chao 1 (Magurran, 1988; Chao and Bunge, 2002) indices of these samples were 620.286-1803.600 (Table 2). The relatively high Shannon and Chao 1 indices suggested that the bacterial diversity in the mariculture farms was high. The bacterial diversity analysis on fish farm sediment indicated that the Shannon index was about 4 and Chao 1 indices ranged from 250 to 2165 (Bissett et al., 2006). The study on bacterial community in the rearing water of Pacific white shrimp showed that the OTUs were in the range of $88-234$, Shannon diversity indices were 2.05-3.90, and Chao 1 indices ranged from 107 to 350 (Zheng et al., 2017). Hua et al. (2017) investigated the bacterial community with different coastal reclamation histories in Jiangsu Province (Eastern China) to find that the Shannon indices and Chao 1 indices were in the ranges of $8-10$ and 2000-20,000, respectively. All studies indicated that the bacterial diversity levels in the intensive fish farms were lower than those in other reclamation coastal ecosystems.

\subsection{Distinct microbial community between conventional and the recirculating mariculture systems}

The conventional intensive mariculture system ( $\mathrm{ZZ} \mathrm{farm)} \mathrm{and} \mathrm{the}$ 

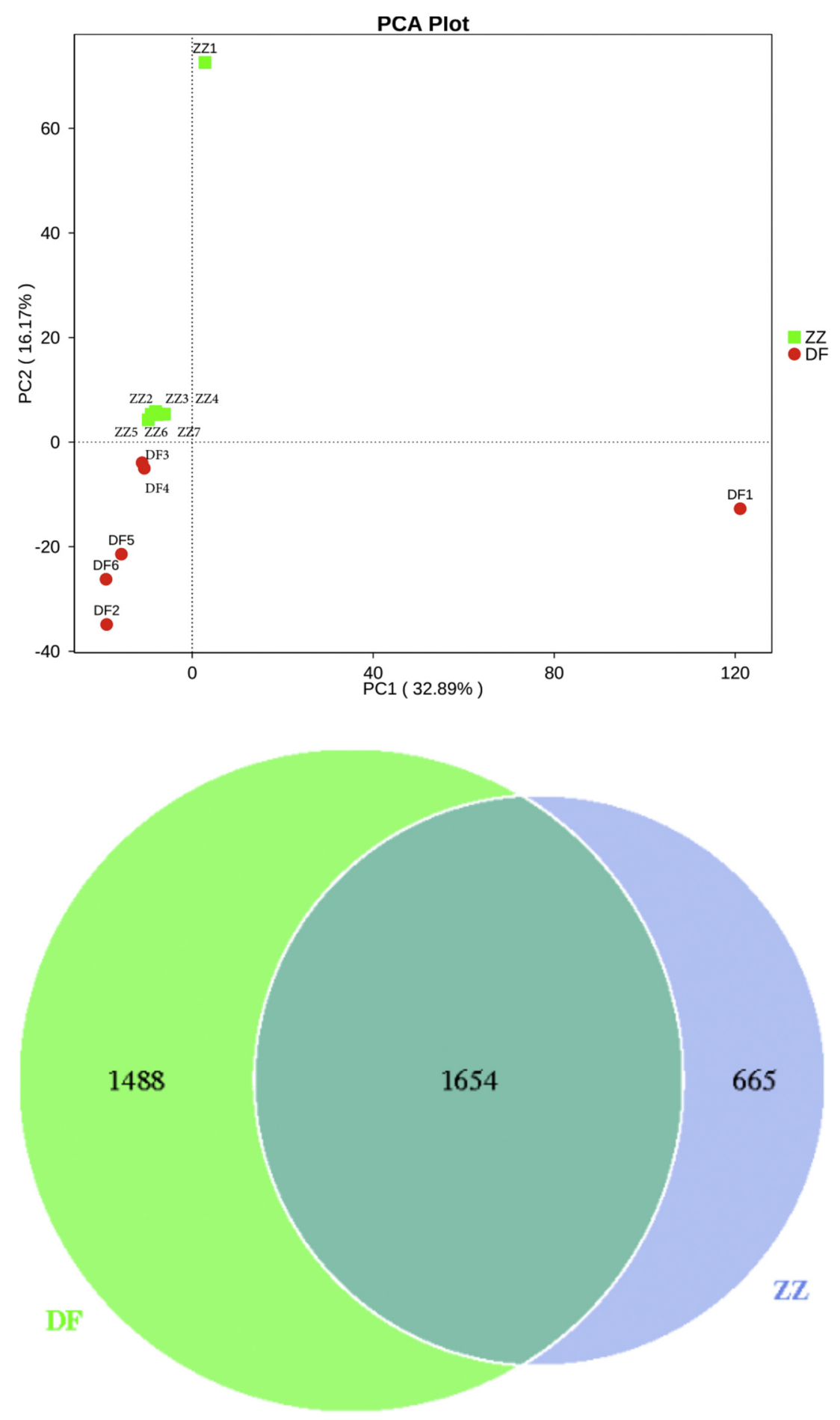

Fig. 2. The principal components analysis (PCA) based on OTU level. The red and green points represent $\mathrm{ZZ}$ and DF samples. ZZ refers to the conventional mariculture system while DF refers to the recirculating mariculture system. (For interpretation of the references to colour in this figure legend, the reader is referred to the web version of this article).
Fig. 3. The common and unique OTUs between ZZ and DF groups displayed by venn graph. Each circle in the figure represents a group: the left circle is for DF, and the right circle is for ZZ. The datum in the overlapped part shows the OTUs number shared by $\mathrm{ZZ}$ and DF. The data in the unoverlapped parts show the unique OTUs number in ZZ or DF group. ZZ refers to the conventional mariculture system while DF refers to the recirculating mariculture system. recirculating mariculture system (DF farm) are two typical aquaculture systems in the coastal zone. The bacterial communities from two different farms were compared at different taxonomic levels. The principal component analysis (PCA) based on OTUs significantly separated the ZZ samples from DF samples, indicating that each farm had its own specific characteristic microbial community, although the influents of two farms were both from the groundwater in the same coastal area (Fig. 2). The venn diagram (Fig. 3) directly showed the similarities and differences between $\mathrm{ZZ}$ and DF farms at OTU level. There were 1654 similar OTUs in the two farms, 1488 different OTUs in DF farm, and 665 different OTUs in ZZ farm. The results showed that the bacteria diversity in $\mathrm{DF}$ farm was richer than that in $\mathrm{ZZ}$ farm, which indicated that recirculating mariculture system led to a greater accumulation of diverse bacteria. Additionally, the influences of environmental variables on the microbial communities of each mariculture system were quite different. Redundancy analysis (RDA) was performed to identify the correlations between taxonomic composition at OTU level and environmental variables (shown in Table 3) such as phosphate (P), total phosphorus (TP), ammonium $\left(\mathrm{NH}_{3}\right)$, nitrate $\left(\mathrm{NO}_{3}\right)$, nitrite $\left(\mathrm{NO}_{2}\right)$, silicate (Si), and total carbon (TOC) (Fig. 4). The first two axes explained $52.09 \%$ of the taxonomic information. RDA analysis illustrated that ZZ samples was positively correlated with $\mathrm{P}, \mathrm{Si}$ and TOC while DF samples were positively correlated with $\mathrm{NH}_{3}, \mathrm{NO}_{3}, \mathrm{NO}_{2}$ and $\mathrm{TP}$, which confirmed the difference in the microbial community of the conventional 
Table 3

Environmental parameters of each water sample.

\begin{tabular}{|c|c|c|c|c|c|c|c|c|}
\hline Sample & $\mathrm{P}(\mathrm{mg} / \mathrm{L})$ & $\begin{array}{l}\mathrm{NH}_{3} \\
(\mathrm{mg} / \mathrm{L})\end{array}$ & $\begin{array}{l}\mathrm{NO}_{2} \\
(\mathrm{mg} / \mathrm{L})\end{array}$ & $\begin{array}{l}\mathrm{NO}_{3} \\
(\mathrm{mg} / \mathrm{L})\end{array}$ & $\begin{array}{l}\text { TP } \\
(\mathrm{mg} / \mathrm{L})\end{array}$ & $\begin{array}{l}\mathrm{TN} \\
(\mathrm{mg} / \mathrm{L})\end{array}$ & $\begin{array}{l}\text { TOC } \\
(\mathrm{mg} / \mathrm{L})\end{array}$ & $\begin{array}{l}\mathrm{Si} \\
(\mathrm{mg} / \mathrm{L})\end{array}$ \\
\hline ZZ1 & 0.01 & 0.02 & 0.00 & 0.48 & 0.01 & 0.63 & 5.36 & 5.05 \\
\hline ZZ2 & 0.12 & 0.15 & 0.01 & 1.44 & 0.01 & 2.20 & 8.03 & 5.22 \\
\hline ZZ3 & 0.01 & 0.15 & 0.01 & 1.48 & 0.01 & 2.00 & 7.44 & 6.13 \\
\hline ZZ4 & 0.00 & 0.18 & 0.01 & 1.41 & 0.02 & 1.92 & 7.49 & 6.38 \\
\hline ZZ5 & 0.10 & 0.16 & 0.01 & 1.62 & 0.01 & 2.00 & 6.88 & 5.11 \\
\hline ZZ6 & 0.10 & 0.14 & 0.01 & 1.48 & 0.01 & 1.87 & 6.92 & 5.00 \\
\hline ZZ7 & 0.00 & 0.02 & 0.00 & 0.05 & 0.02 & 1.65 & 7.43 & 0.23 \\
\hline DF1 & 0.01 & 0.02 & 0.00 & 0.60 & 0.02 & 0.64 & 5.56 & 2.81 \\
\hline DF2 & 0.01 & 0.16 & 0.01 & 3.65 & 0.02 & 3.89 & 6.29 & 2.65 \\
\hline DF3 & 0.03 & 0.17 & 0.01 & 3.52 & 0.04 & 3.67 & 9.6 & 2.45 \\
\hline DF4 & 0.03 & 0.24 & 0.05 & 3.50 & 0.04 & 3.78 & 2.23 & 2.44 \\
\hline DF5 & 0.03 & 0.29 & 0.04 & 1.99 & 0.04 & 3.87 & 5.62 & 2.37 \\
\hline DF6 & 0.03 & 0.28 & 0.03 & 0.47 & 0.04 & 3.56 & 5.21 & 2.43 \\
\hline
\end{tabular}

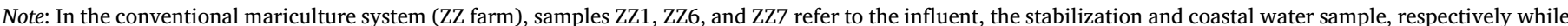

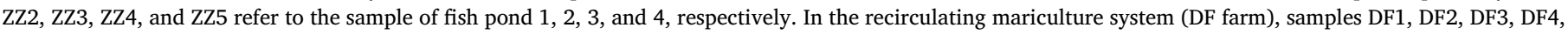

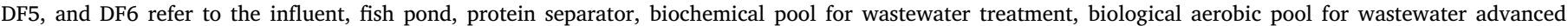
treatment, and the recycled water. $\mathrm{P}$ means phosphate.

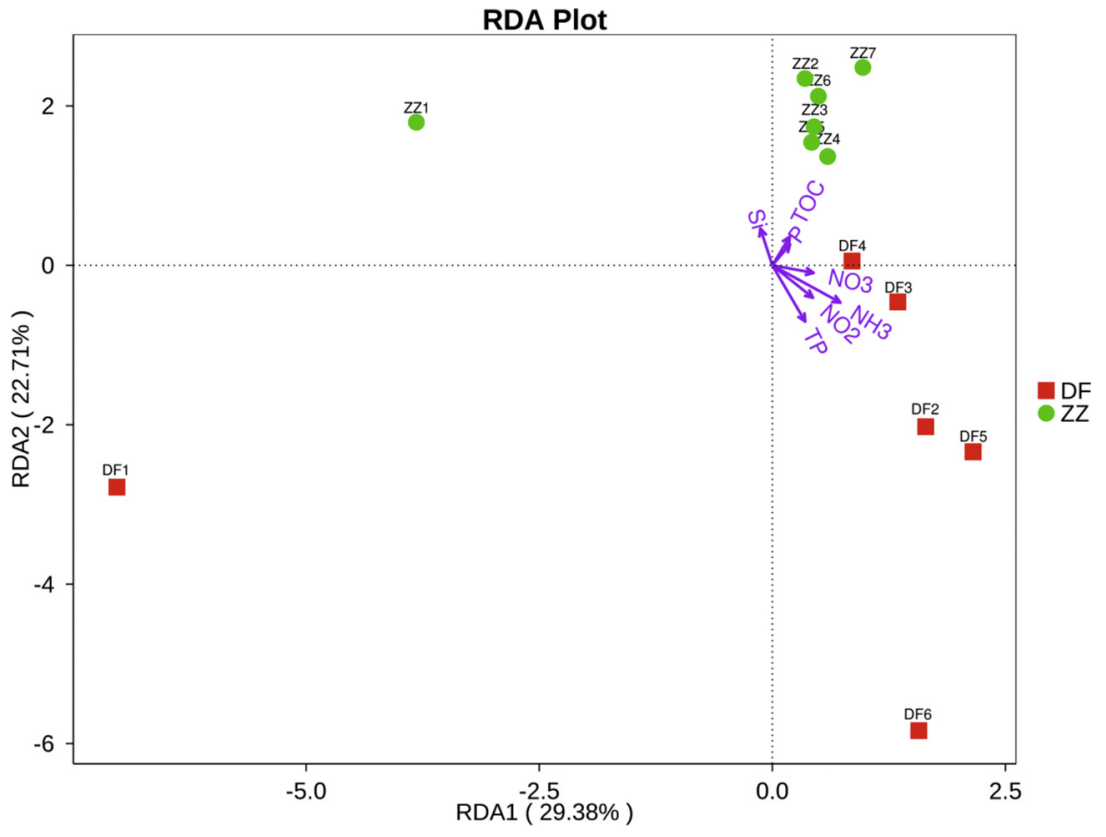

Fig. 4. Redundancy analysis shows the relationships between environmental variables and each sample based on OTU level. Abbreviations: Pphosphate; TPtotal phosphorus; NH3ammonium nitrogen; NO3nitrate nitrogen; NO2nitrite nitrogen; Sisilicate; TOCtotal organic carbon. In the conventional mariculture system (ZZ farm), samples ZZ1, ZZ6, and ZZ7 refer to the influent, the stabilization and coastal water sample, respectively while ZZ2, ZZ3, ZZ4, and ZZ5 refer to the sample of fish pond $1,2,3$, and 4 , respectively. In the recirculating mariculture system (DF farm), samples DF1, DF2, DF3, DF4, DF5, and DF6 refer to the influent, fish pond, protein separator, biochemical pool for wastewater treatment, biological aerobic pool for wastewater advanced treatment, and the recycled water.

and the recirculating mariculture systems. The microbial community in the recirculating system (DF farm) relied on the nitrogen nutrient. Linear discriminant analysis Effect Size (LEfSe) was used to determine the potential discriminating taxa between $\mathrm{DF}$ and $\mathrm{ZZ}$ farms. The results showed that there were 10 bacterial taxa distinguishing DF from $\mathrm{ZZ}$ farm with LDA value greater than 4.0 (Fig. 5a). In ZZ farm, 1 phylum and 1 genus were enriched, including Bacteroidetes (phylum level) and Pseudofulvibacter (genus level) while 1 phylum, 1 order, 1 family, 3 genera and 2 species were enriched in DF farm, including Proteobacteria (phylum level), Vibrionales (from order to species levels), Sulfitobacter (from genus to species levels), and Alteromonas (genus level) (Fig. 5a and b).

The relative abundances of the top 10 bacteria at phylum and genus levels in the two farms were showed in Fig. 6a and b. Fig. 6a showed that the most dominant phyla were Proteobacteria and Bacteroidetes, with the total percentages of the two phyla exceeding $90 \%$ in both farms. The relative abundance of Proteobacteria phylum in DF farm was higher than that in $\mathrm{ZZ}$ farm while the relative abundance of Bacteroidetes phylum in ZZ farm was higher than that in DF farm. Many studies on the structure of microbial community in fish farms had shown that Proteobacteria and Bacteroidetes were the most dominant phyla. Xiong et al. (2015) found that Proteobacteria and Bacteroidetes were the dominant phyla in aquaculture environment of Guangdong, China. Colombo et al. (2016) also found Proteobacteria was the dominant phylum in aquaculture facility. Comparing the genera with high relative abundance (top 10) in the two farms (Fig. 6b), Pseudofulvibacter, Polaribacter, Glaciecola, Crocinitomix, Colwellia, Oleispira and Balneola possessed higher abundance in $\mathrm{ZZ}$ farm while Vibrio, Alteromonas and Pseudoalteromonas showed higher abundance in DF farm. The Pseudofulvibacter and Vibrio were the most dominant genus in ZZ and DF farm, respectively, reconfirming the difference in the microbial community of the conventional and the recirculating mariculture systems. Zheng et al. (2017) found Enterobacter and some unclassified genera of Enterobacteriaceae were the most abundant genera in shrimp at all growth stages, followed by an unclassified genus of Rhodobacteraceae, and then genera Ruegeria, Aquimarina, and Vibrio. Iijima et al. (2009) isolated several strains of Pseudoalteromonas, Vibrio and Halomonas from fish farm sediments in Japan. Bacillus, Vibrio, Acinetobacter, Pseudoalteromonas, Aeromonas, Pseudomonas, Flavobacterium, Edwardsiella and Hafnia were also found in the mariculture 


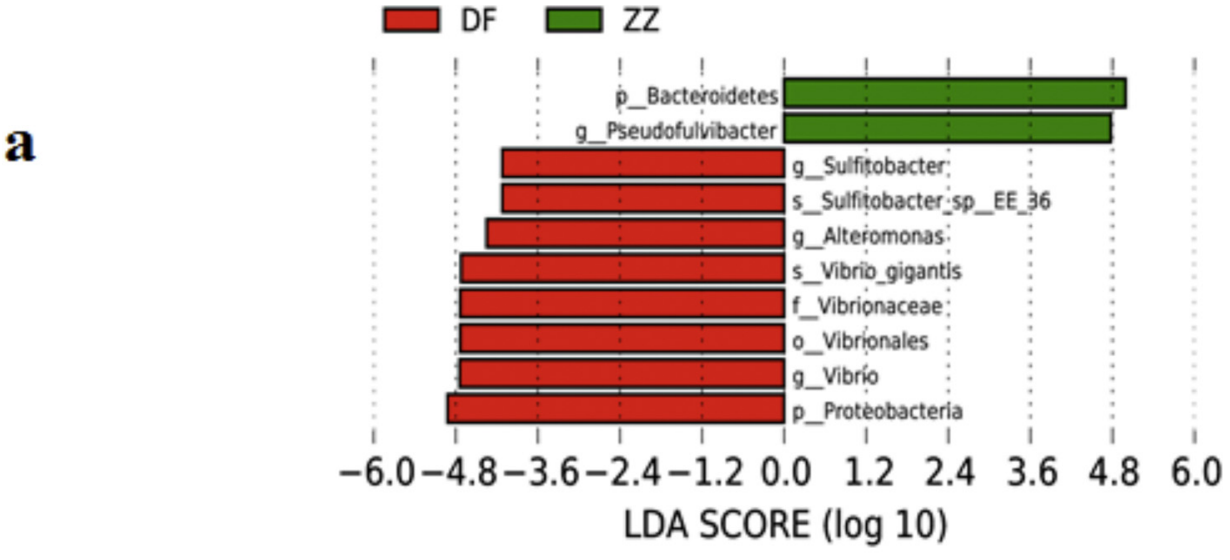

Fig. 5. (a) Linear discriminate analysis (LDA) value of the taxa in the conventional ( $\mathrm{ZZ} \mathrm{farm)}$ and recirculating (DF farm) mariculture systems. The length of the column represents the effect size of the bacterial lineages. (b) The cladograms of bacterial lineages with significant difference between ZZ and DF. The bacterial groups from phylum to species level are listed from center to outside. Each circle's diameter is proportional to the bacterial taxon's abundance. Green: bacterial taxa enriched in ZZ, Red: bacterial taxa enriched in DF. (For interpretation of the references to colour in this figure legend, the reader is referred to the web version of this article).

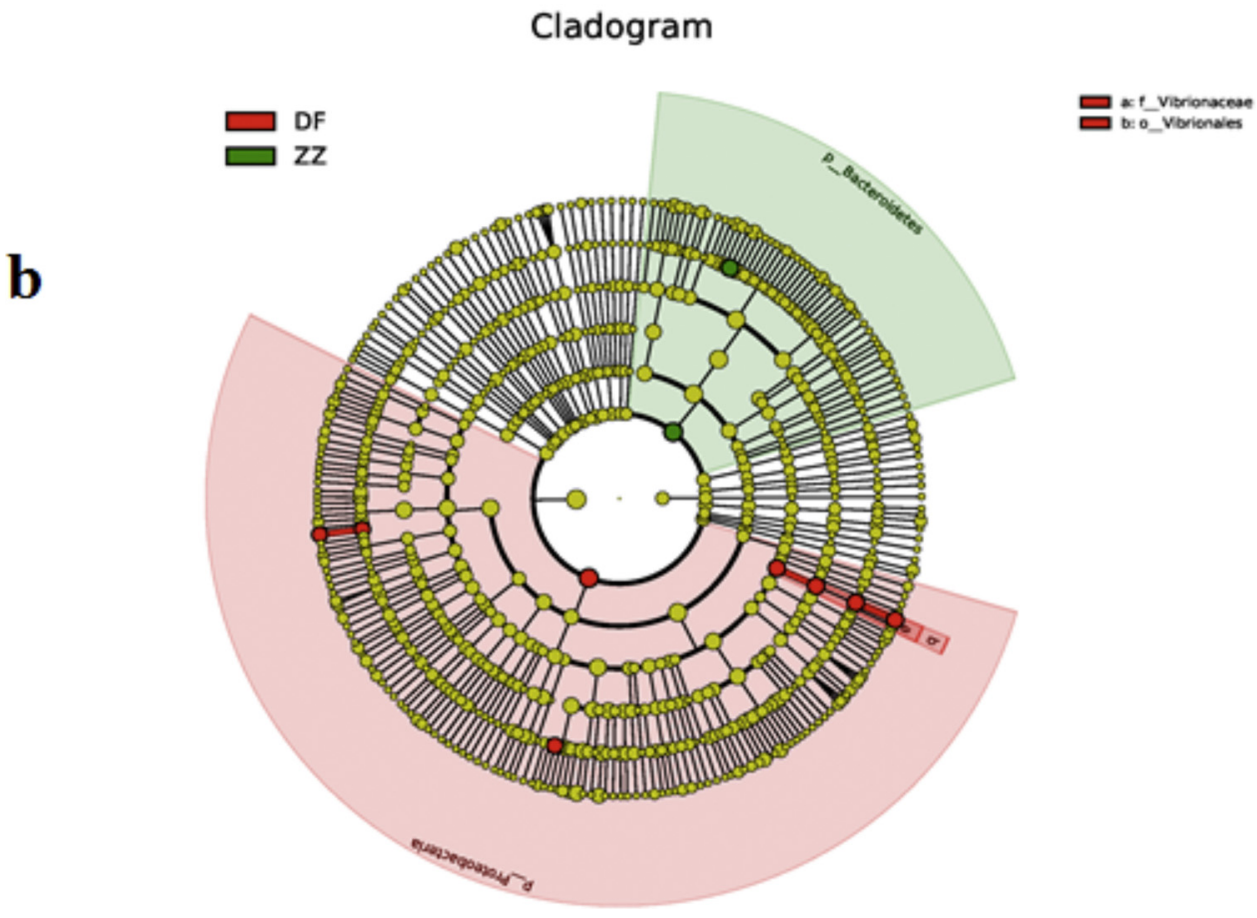

environment in China, Vietnam, and Australia (Akinbowale et al., 2006; Hoa et al., 2008; Gao et al., 2012).

\subsection{Effects of different fish ponds and wastewater treatment units on the} microbial communities

Bacterial communities along with different fish ponds were analyzed to study the variation among different mariculture animals and to find out the fish-specific groups. Compared with ZZ1, the relative abundance of bacteria genera Pseudofulvibacter, Polaribacter, Glaciecola, Crocinitomix, Oleispira and Colwellia decreased whereas that of the bacteria genera Balneola, Phaeodactylibacter, Maritimibacter and Owenweeksia increased in ZZ2 (Fig. 7a). It suggested that the aquaculture fish $P$. vannamei in Pond 1 could induce the propagation of Balneola, Phaeodactylibacter, Maritimibacter and Owenweeksia, and the fish pond might be suitable for the growth of these genera. Compared with ZZ1, the relative abundance of bacteria genera Pseudofulvibacter, Polaribacter, Crocinitomix and Colwellia increased in ZZ3, and the relative abundance of Pseudofulvibacter, Polaribacter, Glaciecola and Colwellia increased in ZZ4 and the relative abundance of Pseudofulvibacter, Crocinitomix and Colwellia increased in ZZ5. Similarly, it indicated that Ponds 2, 3 and 4 were suitable for the target bacteria. The relative abundance of bacteria including Vibrio, Alteromonas, Pseudofulvibacter,
Polaribacter, Pseudoalteromonas, Crocinitomix and Sulfitobacter in sample DF2 that was collected from Pond of $S$. salar increased by comparing the recycled water (DF6) of DF farm (Fig. 7b), which suggested that these bacteria were suitable for $S$. salar culturing. The dominant genera (the relative abundance of the bacteria over $1 \%$ ) in ZZ2 (Pond for $P$. vannamei) were Balneola, Phaeodactylibacter; Maritimibacter and Owenweeksia. The dominant genera in ZZ3 (Pond for S. maximus) were Pseudofulvibacter, Polaribacter, Crocinitomix, Oleispira and Colwellia. The dominant genera in ZZ4 were Pseudofulvibacter, Polaribacter, Glaciecola, Oleispira and Colwellia. The dominant genera in ZZ5 were Pseudofulvibacter, Crocinitomix, Oleispira and Colwellia while those in DF2 were Vibrio, Alteromonas, Polaribacter, Pseudoalteromonas, Colwellia, and Sulfitobacter (Fig. 7). The investigation indicated that characteristics of the microbial community in the fish farms were dependent on the cultured animals. Study in Vietnamese shrimp ponds showed that Vibrio and Bacillus were the dominant bacteria (Hoa et al., 2008). Colombo et al. (2016) studied the bacteria community of water from a salmon farm in Italy and found Bacillus, Synechococcus, Mycobacterium, Enterobacteria, Pseudomonas, Cellulophoga, Vibrio, Burkholderia, Escherichia, and Ralstonia were the top 10 genera. Predominant bacteria in the salmonid fish-farm in Australia included Sphyngopyxis, Rhodobacter, Nitrospira, Desulfosarcina, Colwellia, Halomonas, Moritella, Oceanospirillium, Photobacterium, and Pseudoalteromonas (Bissett et al., 2005). Eight different 


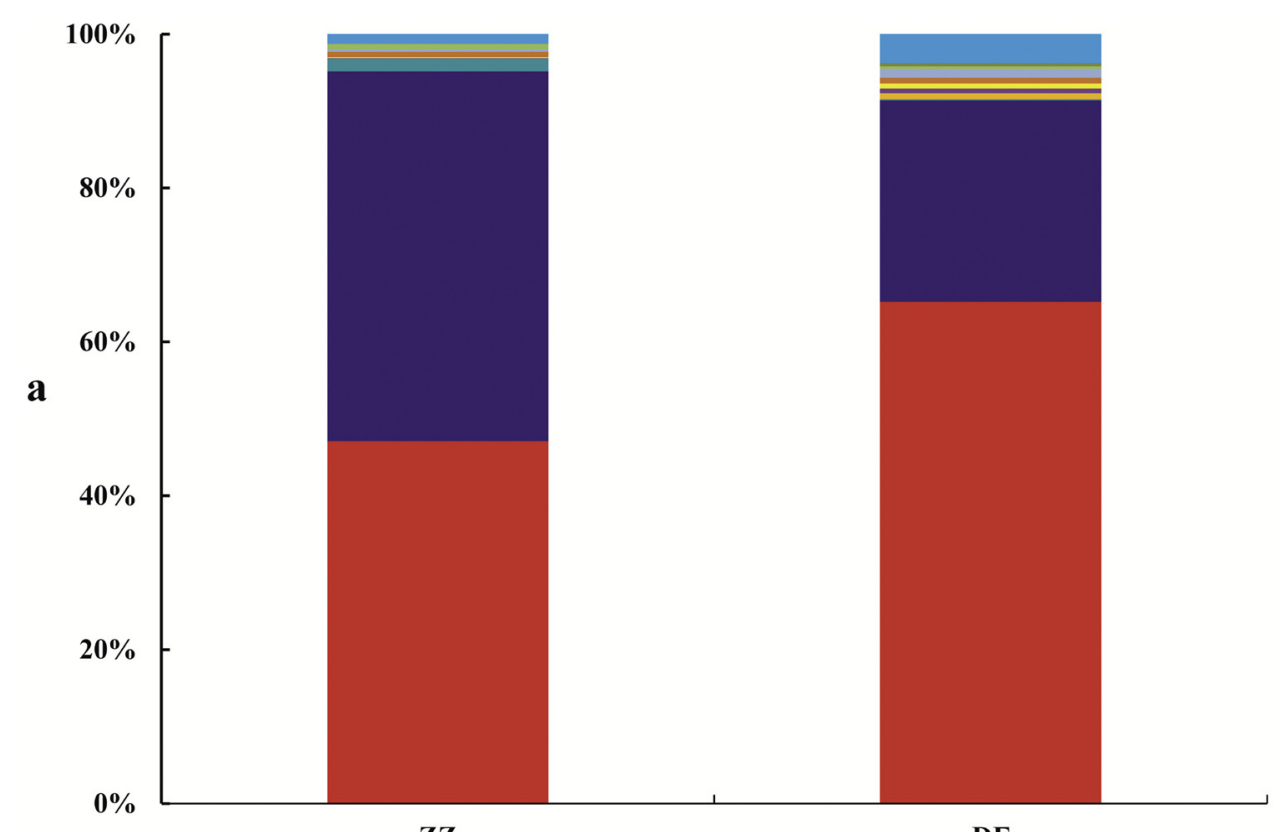

$\mathbf{Z Z}$

DF
- Proteobacteria

- Cyanobacteria

- Parcubacteria

- Verrucomicrobia

Gracilibacteria

- Others
- Bacteroidetes

Spirochaetes

Firmicutes

Planctomycetes

-Woesearchaeota_.DHVEG-6.

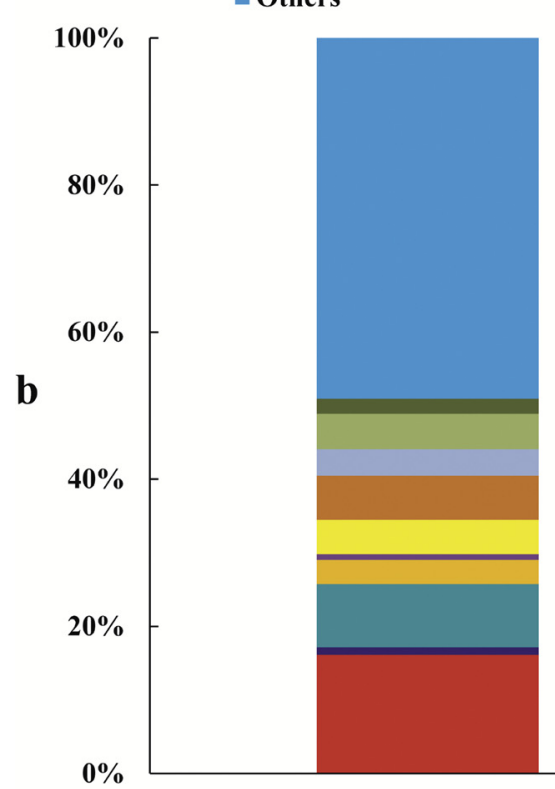

ZZ

- Pseudofulvibacter Glaciecola - Colwellia - Balneola

\section{- Vibrio \\ Alteromonas Pseudoalteromonas - Others}

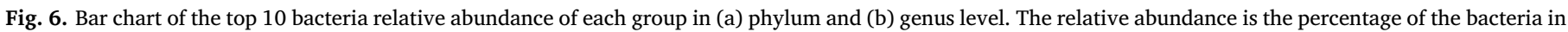
total microorganism. ZZ refers to the conventional mariculture system while DF refers to the recirculating mariculture system.

genera including Acinetobacter, Aeromonas, Chryseobacterium, Erwinia, Flavobacterium, Pseudomonas and Rheinheimera were identified in the salmonid fish farm in Northern Ireland (Moore et al., 2014). Enger et al. (1989) isolated Vibrio from the sediments of fish farm on the western coast of Southern Norway. The bacterial groups also exhibited growth stage-specific features. Relative abundances of Pseudofulvibacter and Crocinitomix in ZZ3 (Pond for adult $S$. maximus) were higher than those in ZZ4 (Fig. 7a). However, relative abundances of Polaribacter,
Glaciecola, Oleispira and Colwellia in ZZ4 (Pond for fish-fry S. maximus) were higher than those in ZZ3. Zheng et al. (2017) also found that growth stages made effect on the bacterial communities of Pacific White Shrimp (Litopenaeus vannamei) in rearing water. Rhodobacteraceae was abundant in rearing water at all tested growth stages whereas its relative abundance displayed a decreasing trend at mysis and postlarva stages (Zheng et al., 2017). Flavobacteriaceae was abundant at zoea stage compared with that at mysis stage (Zheng et al., 2017). At 


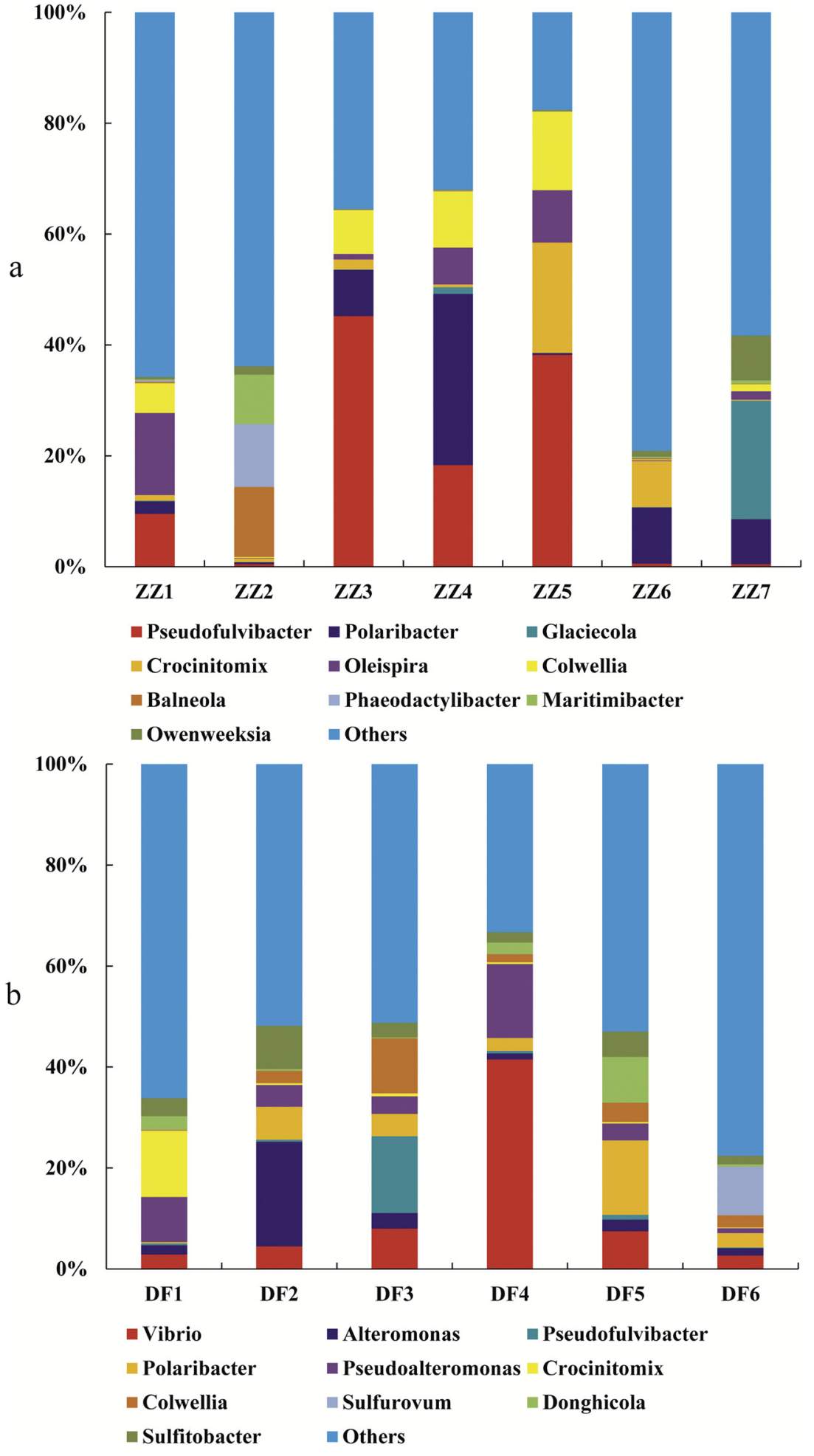

Fig. 7. Bar chart of the top 10 bacteria relative abundance of each sample from ZZ farm (a) and DF farm (b) in genus level. The relative abundance is the percentage of the bacteria in total microorganism. ZZ refers to the conventional mariculture system while DF refers to the recirculating mariculture system. In the conventional mariculture system ( $Z Z$ farm), samples ZZ1, ZZ6, and ZZ7 refer to the influent, the stabilization and coastal water sample, respectively while ZZ2, ZZ3, ZZ4, and ZZ5 refer to the sample of fish pond 1, 2, 3, and 4, respectively. In the recirculating mariculture system (DF farm), samples DF1, DF2, DF3, DF4, DF5, and DF6 refer to the influent, fish pond, protein separator, biochemical pool for wastewater treatment, biological aerobic pool for wastewater advanced treatment, and the recycled water. postlarva stage, Microbacteriaceae (phylum Actinobacteria) increased to become the dominant bacterial group (Zheng et al., 2017).

Bacterial communities along with different wastewater treatment stages were analyzed to find out the stage-specific groups. Variation of bacterial community in the samples collected from DF farm was observed along with the wastewater treatment stages (Fig. 7b). The relative abundances of Vibrio, Pseudofulvibacter, Crocinitomix and Colwellia in sample DF3 were significantly higher than those in DF2, indicating the accumulation of these bacteria in the protein separation process. However, the relative abundances of Alteromonas, Polaribacter,
Pseudoalteromonas and Sulfitobacter in DF3 were much lower than those in DF2, suggesting that the protein separation process could remove these bacteria efficiently. The relative abundances of Vibrio, Pseudoalteromonas and Donghicola sharply increased in DF4 when compared with samples DF1 and DF3, indicating that these bacteria were accumulated in this biochemical water treatment process. It was found that the relative abundances of Alteromonas, Pseudofulvibacter, Polaribacter, Colwellia, Donghicola and Sulfitobacter accumulated in the aerobic process (DF5) when comparing samples DF5 and DF4. All of the bacteria (TOP 10 shown in Fig. 7b) in DF6 were reduced much in comparison 
with DF5, indicating that UV could make removal effect on these bacteria. In addition, in contrast to DF1, it was found that many bacteria such as Pseudofulvibacter, Sulfitobacter, Crocinitomix, Pseudoalteromonas and Donghicola could be removed efficiently by the treatment processes in DF farm. These results indicated that the bacterial groups exhibited treatment stage-specific features, and the wastewater treatment systems used in the recirculating system (DF farm) could remove bacteria effectively. Disinfection process, such as chlorine disinfection and ultraviolet disinfection, was the main technique among many wastewater treatment methods to eliminate microbes especially bacteria (Shi et al., 2013; Wen et al., 2016). The microbial removal efficiency of other treatment processes including activated sludge process was relatively low (Yang et al., 2014; Wen et al., 2016).

\subsection{Potential pathogens in the intensive mariculture systems and coastal waters}

Some bacteria detected in fish farms or coastal waters were found as the pathogens such as Aeromonas, Acinetobacter, Arcobacter, Clostridium, Flavobacterium, Vibrio, Yersinia, Pseudoalteromonas, and Pseudomonas, and Shewanella (Enger et al., 1989; Viljamaa-Dirks, 2016; Xiong et al., 2015; Lamb et al., 2017). Potential pathogens including Vibrio, Arcobacter, Pseudoalteromonas, and Shewanella were detected in the mariculture systems (Fig. 8). Worst of all, Vibrio, Arcobacter, Pseudoalteromonas, and Shewanella were found as the dominant genus in the recirculating mariculture (DF) farm, indicating that potential pathogens could become the majority of microorganisms in the intensive mariculture systems even in the absence of recorded fish diseases. The Vibrio spp. was common pathogen which occurred only in sea farms (Viljamaa-Dirks, 2016). It was reported that high abundance of Gamma-proteobacteria presented in diseased shrimps was attributed to Vibrio (Rungrassamee et al., 2016). In the mariculture farm of Pacific White Shrimp (Litopenaeus vannamei), Vibrio was also found as one of the dominant genera in shrimp (Zheng et al., 2017). Another study on Vietnamese shrimp ponds reported that Vibrio was a common antibiotic resistance pathogen (Hoa et al., 2008). Xiong et al. (2015) found that fish ponds were reservoirs of pathogens related to human health such as Acinetobacter, Arcobacter, and Clostridium.

Mariculture is a major industry in global coastal zones and has led to concerns about the impact of fish farming on marine environments. In order to evaluate the effect of the mariculture farm on the coastal waters, the microbial community of the sample ZZ7 collected from coastal marine near a lot of fish farms including ZZ and DF farms was investigated. Comparing ZZ7 with samples collected from ZZ and DF farms, the potential pathogens genera such as Vibrio, Arcobacter, Pseudoalteromonas, and Shewanella detected in the coastal mariculture

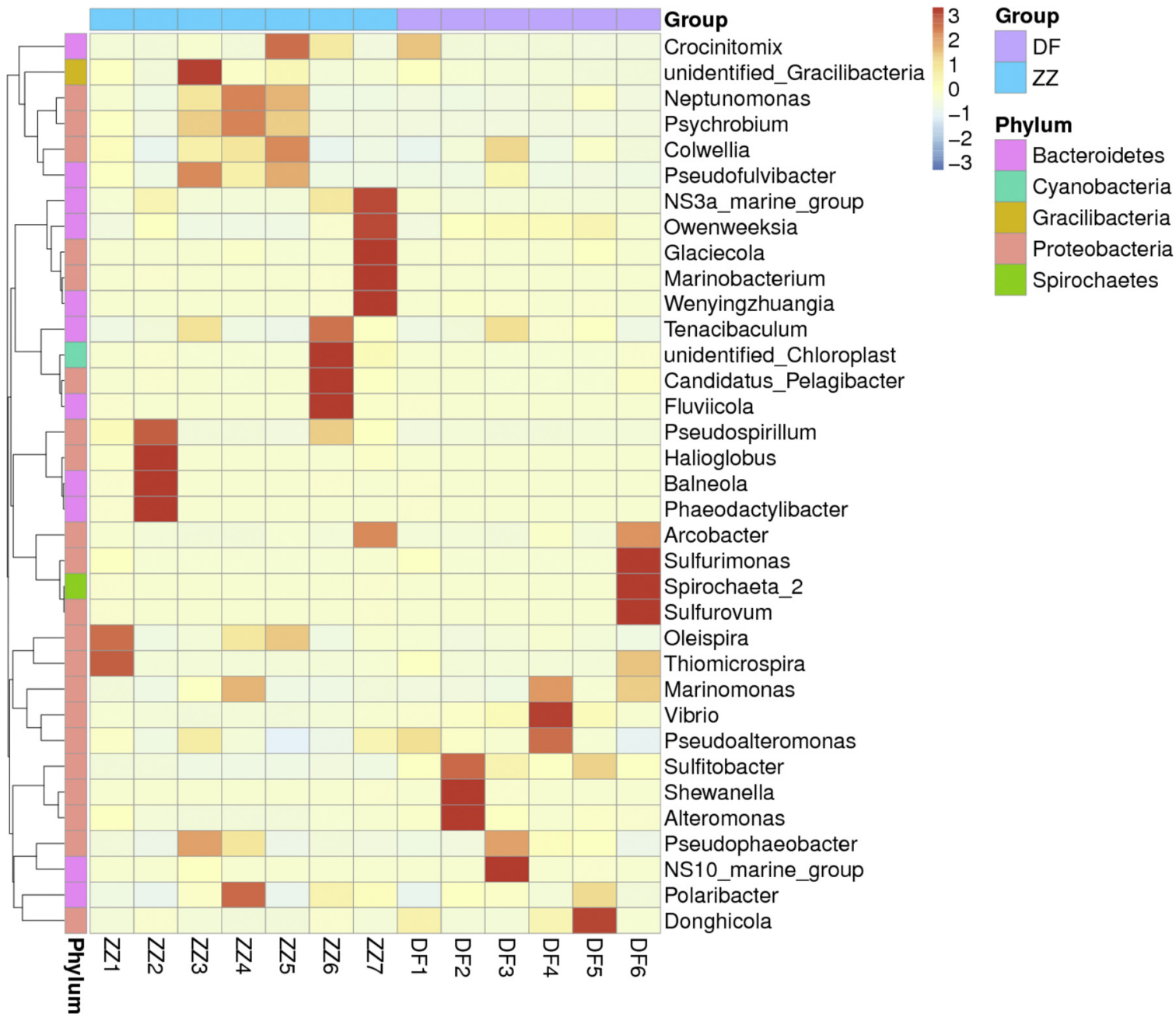

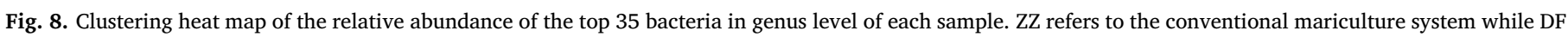

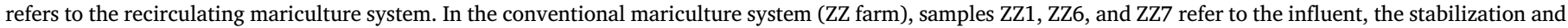

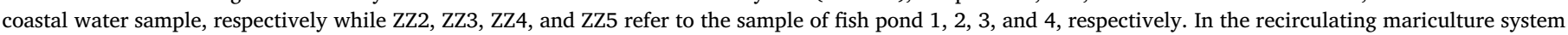

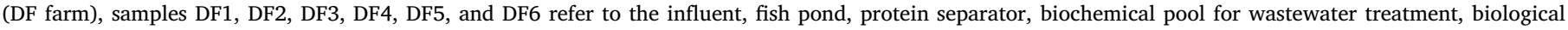
aerobic pool for wastewater advanced treatment, and the recycled water. 
systems were also found in the adjacent coastal water (ZZ7) (Fig. 8). Among these potential pathogens, Arcobacter was found as a dominant genus in the coastal waters near the mariculture farms (Fig. 8). The comparison results suggested that the discharge of tail water from intensive mariculture system could affect the microbial community of coastal waters and bring potential harmful bacteria (Fig. 8). Harnisz et al. (2015) studied the impact of a freshwater fish farm and found that the fish farm affected water quality and increased the diversity of tetracycline-resistance bacteria in the river near the farm. The detection of potential pathogens suggested that the coastal intensive mariculture systems were the hotspot of pathogens which might exert the potential risks to both human and fish health.

\section{Conclusions}

The structural profiles of microbial community in the typical conventional and recirculating mariculture farms were investigated using high-throughput sequencing technology. Each farm had its own microbial community characteristics. Among the top 10 genera in coastal intensive mariculture systems, the predominant bacteria in conventional intensive mariculture system were the Pseudofulvibacter, Polaribacter, Glaciecola, Crocinitomix, Colwellia, Oleispira and Balneola while those in the recirculating systems were Vibrio, Alteromonas and Pseudoalteromonas. The bacteria community structure in the fish pond relied on the mariculture animals. Potential pathogen such as Vibrio, Arcobacter, Pseudoalteromonas, and Shewanella were readily accumulated as dominant bacteria in the recirculating mariculture system (Fig. 8). The detection of similar potential pathogens in the mariculture systems and the adjacent coastal water indicated that the mariculture farms without recirculation system might be the hotspot of pathogens and have great influences on the surrounding coastal waters. The discharge of tail water from mariculture farm had an impact on the microbial community of coastal waters. The wastewater treatment processes used in the recirculating mariculture system displayed a high removal efficiency on bacteria, indicating that the recirculating mariculture system may be more environmentally friendly than the conventional mariculture system.

\section{Acknowledgements}

This work was financially supported by National Natural Science Foundation of China (No. 41671319), One Hundred Talents Program of Chinese Academy of Sciences (Grant numbers of Y629041021 and Y610061033), Two-Hundred Talents Plan of Yantai (Y739011021), CAS Key Research Program of Frontier Sciences (No. QYZDJ-SSWDQC015), and Research Program of CAS Key Laboratory of Coastal Environmental Processes and Ecological Remediation (No. 1189010002).

\section{References}

Akinbowale, O.L., Peng, H., Barton, M.D., 2006. Antimicrobial resistance in bacteria isolated from aquaculture sources in Australia. J. Appl. Microbiol. 100, 1103-1113.

Antunes, P., Gil, O., 2004. PCB and DDT contamination in cultivated and wild sea bass from Ria de Aveiro, Portugal. Chemosphere 54, 1503-1507.

Armougom, F., Raoult, D., 2009. Exploring microbial diversity using 16S rRNA highthroughput methods. J. Comput. Sci. Syst. Biol. 2, 74-92.

Bissett, A., Bowman, J., Burke, C., 2006. Bacterial diversity in organically-enriched fish farm sediments. FEMS Microbiol. Ecol. 55, 48-56.

Cabello, F.C., 2006. Heavy use of prophylactic antibiotics in aquaculture: a growing problem for human and animal health and for the environment. Environ. Microbiol. $8,1137-1144$.

Caporaso, J.G., Kuczynski, J., Stombaugh, J., et al., 2010. QIIME allows analysis of highthroughput community sequencing data. Nat. Methods 7, 335-336.

Chao, A., Bunge, J., 2002. Estimating the number of species in a stochastic abundance model. Biometrics 58, 531-539.

Colombo, S., Arioli, S., Guglielmetti, S., Lunelli, F., Mora, D., 2016. Virome-associated antibiotic-resistance genes in an experimental aquaculture facility. FEMS Microbiol. Ecol. $92 \mathrm{f}$ iw003.

Edgar, R.C., Haas, B.J., Quince, C., Knight, R., 2011. UCHIME improves sensitivity and speed of chimera detection. Bioinformatics 27, 2194-2200.

Enger, Ø., Husevag, B., Goksøyr, J., 1989. Presence of the fish pathogen Vibrio salmonicida in fish farm sediments. Appl. Environ. Microbiol. 55, 2815-2818.

FAO, 2009. State of World Aquaculture 2008. Available at:. Fisheries and Aquaculture Department, Food and Agriculture Organization of the United Nations, Rome. http:// www.fao.org/docrep/011/i0250e/i0250e00.HTM.

Gao, P., Mao, D., Luo, Y., Wang, L., Xu, B., Xu, L., 2012. Occurrence of sulfonamide and tetracycline-resistant bacteria and resistance genes in aquaculture environment. Water Res. 46, 2355-2364.

Harnisz, M., Korzeniewska, E., Golaš, I., 2015. The impact of a freshwater fish farm on the community of tetracycline-resistant bacteria and the structure of tetracycline resistance genes in river water. Chemosphere 128, 134-141.

Heuer, O.E., Kruse, H., Grave, K., Collignon, P., Karunasagar, I., Angulo, F.J., 2009. Human health consequences of use of antimicrobial agents in aquaculture. Clin. Infect. Dis. 49, 1248-1253.

Hoa, P.T.P., Nonaka, L., Viet, P.H., Suzuki, S., 2008. Detection of the sul1, sul2, and sul3 genes in sulfonamide-resistant bacteria fromwastewater and shrimp ponds of north Vietnam. Sci. Total Environ. 405, 377-384.

Holmer, M., Wildish, D., Hargrave, B.T., 2005. Organic enrichment from marine finfish aquaculture and effects on sediment biogeochemical processes. In: Hargrave, B.T. (Ed.), Environmental Effects of Marine Finfish Aquaculture. Springer, Berlin, pp. 181-206.

Hua, J., Feng, Y., Jiang, Q., Bao, X., Yin, Y., 2017. Shift of bacterial community structure along different coastal reclamation histories in Jiangsu, Eastern China. Sci. Rep. 7, 10096.

Iijima, S., Washio, K., Okahara, R., Morikawa, M., 2009. Biofilm formation and proteolytic activities of Pseudoalteromonas bacteria that were isolated from fish farm sediments. Microb. Biotechnol. 2, 361-369.

Kalantzi, I., Karakassis, I., 2006. Benthic impacts of fish farming: metaanalysis of community and geochemical data. Mar. Pollut. Bull. 52, 479-483.

Lamb, J.B., van de Water, J.A.J.M., Bourne, D.G., Altier, C., Hein, M.Y., Fiorenza, E.A., Abu, N., Jompa, J., Harvell, C.D., 2017. Seagrass ecosystems reduce exposrure to bacterial pathogens of humans, fishes, and invertebrates. Science 355, 731-733.

Leite, A.M., Mayo, B., Rachid, C.T., Pexoto, R.S., Silva, J.T., Paschoalin, V.M., Delqado, S. 2012. Assessment of the microbial diversity of Brazilian kefir grains by PCR-DGGE and pyrosequencing analysis. Food Microbiol. 31, 215-221.

Magurran, A.E., 1988. Ecological Diversity and Its Measurement. Princeton University Press, Princeton, NJ.

Moore, J.E., Huang, J., Yu, P., Ma, C., Moore, P.J., Millar, B.C., Goldsmith, C.E., Xu, J., 2014. High diversity of bacterial pathogens and antibiotic resistance in salmonid fish farm pond water as determined by molecular identification employing $16 \mathrm{~S}$ rDNA PCR, gene sequencing and total antibiotic susceptibility techniques. Ecotoxicol. Environ. Saf. 108, 281-286.

Muziasari, W.I., Pitkänen, L.K., Sørum, H., Stedtfeld, R.D., Tiedje, J.M., Virta, M., 2017. The resistome of farmed fish feces contributes to the enrichment of antibiotic resistance genes in sediments below Baltic Sea fish farms. Front. Microbiol. 7, 2137.

Rungrassamee, W., Klanchui, A., Maibunkaew, S., Karoonuthaisiri, N., 2016. Bacterial dynamics in intestines of the black tiger shrimp and the Pacific white shrimp during Vibrio harveyi exposure. J. Invertebr. Pathol. 133, 12-19.

Sapkota, A., Sapkota, A.R., Kucharski, M., Burke, J., McKenzie, S., Walker, P., Lawrence, R., 2008. Aquaculture practices and potential human health risks: current knowledge and future priorities. Environ. Int. 34, 1215-1226.

Shi, P., Jia, S., Zhang, X.X., Zhang, T., Cheng, S., Li, A., 2013. Metagenomic insights into chlorination effects on microbial antibiotic resistance in drinking water. Water Res. 47, 111-120.

Su, H.C., Ying, G.G., Tao, R., Zhang, R.Q., Fogarty, L.R., Kolpin, D.W., 2011. Occurrence of antibiotic resistance and characterization of resistance genes and integrons in Enterobacteriaceae isolated from integrated fish farms in south China. J. Environ. Monit. 13, 3229-3236.

Tomassetti, P., Persia, E., Mercatali, I., Vani, D., Marussso, V., Porrello, S., 2009. Effects of mariculture on macrobenthic assemblages in a western mediterranean site. Mar. Pollut. Bull. 58, 533-541.

Vezzulli, L., Moreno, M., Marin, V., Pezzati, E., Bartoli, M., Fabiano, M., 2008. Organic waste impact of captured-based Atlantic bluefin tuna aquaculture at an exposed site in the Mediterranean Sea. Estuar. Coast. Shelf Sci. 78, 369-384.

Viljamaa-Dirks, S., 2016. (EVIRA) Katsaus kalaterveyteen 2015. Fish Health Day 2016. Helsinki.

Wang, Q., Garrity, G.M., Tiedje, J.M., Cole, J.R., 2007. Naïve Bayesian classifier for rapid assignment of rRNA sequences into the new bacterial taxonomy. Appl. Environ. Microbiol. 73, 5261-5267.

Wen, Q., Yang, L., Duan, R., Chen, Z., 2016. Monitoring and evaluation of antibiotic resistance genes in four municipal wastewater treatment plants in Harbin, Northeast China. Environ. Pollut. 212, 34-40.

Xiong, W., Sun, Y., Zhang, T., Ding, X., Li, Y., Wang, M., Zeng, Z., 2015. Antibiotics, antibiotic resistance genes, and bacterial community composition in fresh water aquaculture environment in China. Microb. Ecol. 70, 425-432.

Xiong, J., Dai, W., Li, C., 2016. Advances, challenges, and directions in shrimp disease control: the guidelines from an ecological perspective. Appl. Microbiol. Biotechnol. $100,6947-6954$

Yang, Y., Li, B., Zou, S., Fang, H.H.P., Zhang, T., 2014. Fate of antibiotic resistance genes in sewage treatment plant revealed by metagenomic approach. Water Res. 62, 97-106.

Zheng, Y., Yu, M., Liu, J., Qiao, Y., Wang, L., Li, Z., Zhang, X.H., Yu, M., 2017. Bacterial community associated with healthy and diseased Pacific white shrimp (Litopenaeus vannamei) larvae and rearing water across different growth stages. Front. Microbiol. 8, 1362 .

Zhou, Z.C., Zheng, J., Wei, Y.Y., Chen, T., Dahlgren, R.A., Shang, X., Chen, H., 2017. Antibiotic resistance genes in an urban river as impacted by bacterial community and physicochemical parameters. Environ. Sci. Pollut. Res. 24, 23753-23762. 